\title{
A Pyloric Gland-Phenotype Ovarian Mucinous Tumor Resembling Lobular Endocervical Glandular Hyperplasia in a Patient with Peutz-Jeghers Syndrome
}

\author{
Eun Na Kim · Gu-Hwan Kim ${ }^{1}$ \\ Jiyoon Kim · In Ah Park \\ Jin Ho Shin · Yun Chai \\ Kyu-Rae Kim
}

Department of Pathology, Asan Medical Center, University of Ulsan College of Medicine, Seoul; ${ }^{1}$ Department of Medical Genetics, Asan Medical Center Children's Hospital, Seoul, Korea

\author{
Received: April 6, 2016 \\ Revised: June 27, 2016 \\ Accepted: July 1, 2016 \\ Corresponding Author \\ Kyu-Rae Kim, MD \\ Department of Pathology, Asan Medical Center, \\ University of Ulsan College of Medicine, \\ 88 Olympic-ro 43-gil, Songpa-gu, Seoul 05505, \\ Korea \\ Tel: +82-2-3010-4514 \\ Fax: $+82-2-472-7898$ \\ E-mail: krkim@amc.seoul.kr
}

\begin{abstract}
We describe an ovarian mucinous neoplasm that histologically resembles lobular endocervical glandular hyperplasia (LEGH) containing pyloric gland type mucin in a patient with Peutz-Jeghers syndrome (PJS). Although ovarian mucinous tumors rarely occur in PJS patients, their pyloric gland phenotype has not been clearly determined. The histopathologic features of the ovarian mucinous tumor were reminiscent of LEGH. The cytoplasmic mucin was stained with periodic acid-Schiff reaction after diastase treatment but was negative for Alcian blue $\mathrm{pH} 2.5$, suggesting the presence of neutral mucin. Immunohistochemically, the epithelium expressed various gastric markers, including MUC6, HIK1083, and carbonic anhydrase-IX. Multiple ligation-dependent probe amplification detected a germline heterozygous deletion mutation at exons 1-7 of the STK11 gene (c.1-?_920+?del) in peripheral blood leukocytes and mosaic loss of heterozygosity in ovarian tumor tissue. Considering that LEGH and/or gastric-type cervical adenocarcinoma can be found in patients with PJS carrying germline and/or somatic STK11 mutations, our case indicates that STK11 mutations have an important role in the proliferation of pyloric-phenotype mucinous epithelium at various anatomical locations.
\end{abstract}

Key Words: Pyloric gland type; Lobular endocervical glandular hyperplasia; Mucinous; Ovary; PeutzJeghers syndrome
Peutz-Jeghers syndrome (PJS) is an autosomal dominant hereditary disorder characterized by hamartomatous polyposis of the gastrointestinal tract and mucocutaneous pigmentation around the lips. Patients with PJS have a higher incidence of benign or malignant neoplasia of the female genital tract, including minimal deviation adenocarcinoma of the uterine cervix, sex cord tumor with annular tubules, Sertoli cell tumor of the ovary, ${ }^{1}$ and mucinous metaplasia of the fallopian tube and endometrium, as well as extragenital tumors, such as carcinomas of the breast, pancreas, and gastrointestinal tract. Germline mutation of the serine/threonine kinase 11 (STK11)/liver kinase B1 (LKB1) gene in patients with $\mathrm{PJS}^{2}$ and somatic mutation of the STK11 gene in patients with sporadic cases of minimal deviation adenocarcinoma of the uterine cervix ${ }^{3}$ have suggested that the protein product of the STK11 gene has a putative tumor suppressor function and that mutation of the gene plays an important role in the pathogenesis of mucin-producing tumors at various sites. On the other hand, lobular endocervical glandular hyperplasia (LEGH) of the uterine cervix has rarely been reported in patients with PJS; however, ovarian tumor resembling LEGH is even rarer in these patients.

We encountered an ovarian mucinous neoplasm in a patient with PJS (confirmed by STK11 germline mutation), in which the histopathologic features of the ovarian mass were reminiscent of LEGH. The epithelium contained pyloric gland-type mucin and expressed immunohistochemical markers for pyloric glands. Here, we describe the clinicopathologic findings in a PJS patient with an ovarian mucinous tumor with a pyloric gland type mucin.

\section{CASE REPORT}

A 47-year-old postmenopausal woman presented with slowly increasing abdominal girth with a palpable abdominal mass and vaginal bleeding. At the age of 15 , she underwent abdominal 

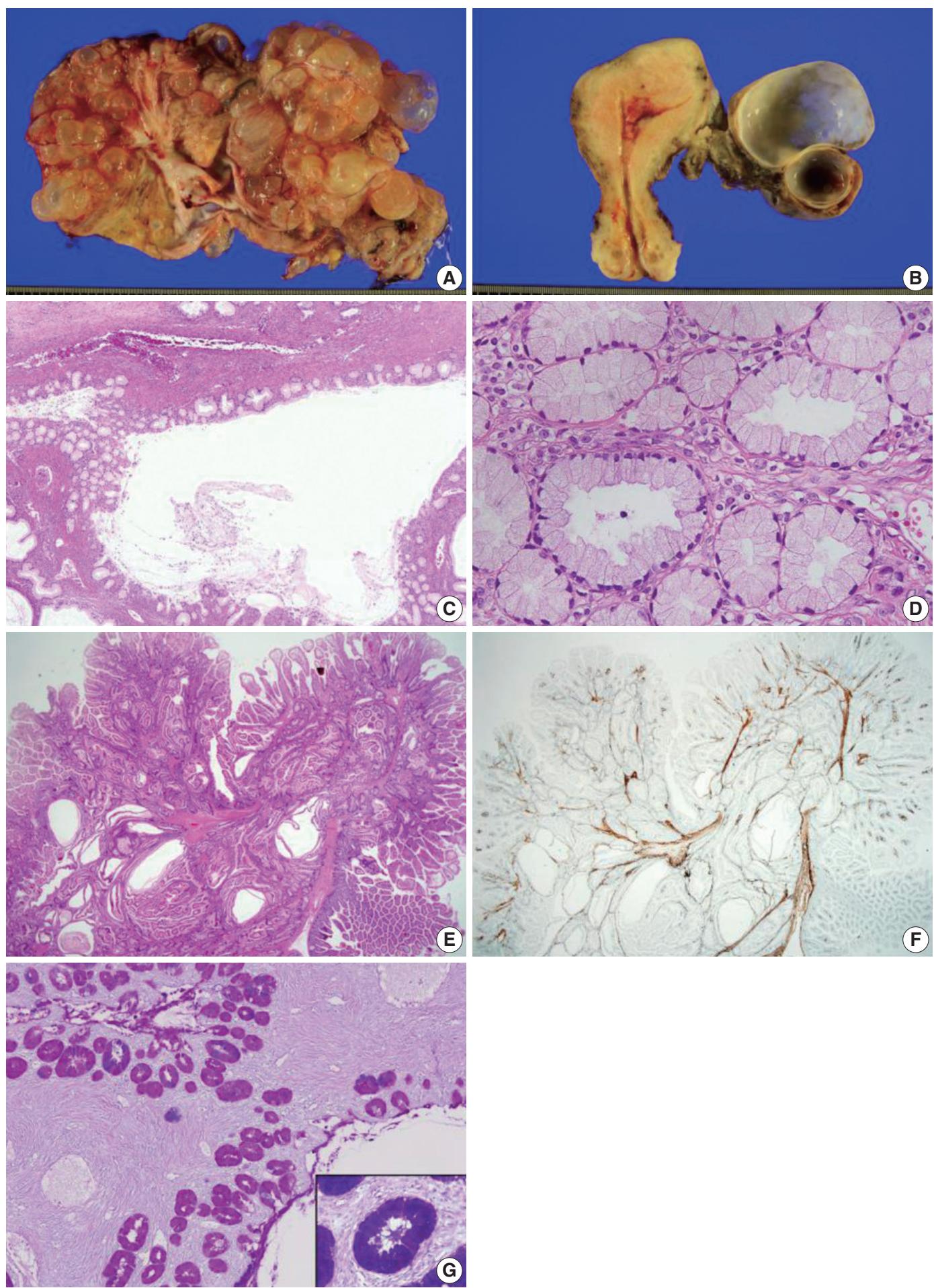

Fig. 1. Sectioned surface of the left ovary showing multilocular cysts filled with mucinous fluid (A) and the cut surface of the right ovary (B). No apparent abnormality is identified on the surface of the uterine cervix and endometrium. Microscopically, the ovarian mucinous tumor is composed of a large dilated duct-like structure with clusters of small glands, reminiscent of lobular endocervical glandular hyperplasia of the uterine cervix (C). At higher magnification, the cysts are lined by a single layer of mucinous epithelium with bland nuclei and pale eosinophilic cytoplasm. Stratification or mitosis is not identified (D). The small bowel polyps in this patient show hyperplastic mucosa with arborizing strands of smooth muscle in various directions (E), highlighted by desmin immunostaining (F). Combined Alcian blue pH 2.5 and periodic acid-Schiff stains after diastase treatment show bright pink-colored cytoplasm, indicating predominant neutral mucin in the cytoplasm of the ovarian mucinous epithelium (G) in contrast to the purple-violet color in normal endocervical epithelium (G, inlet). 

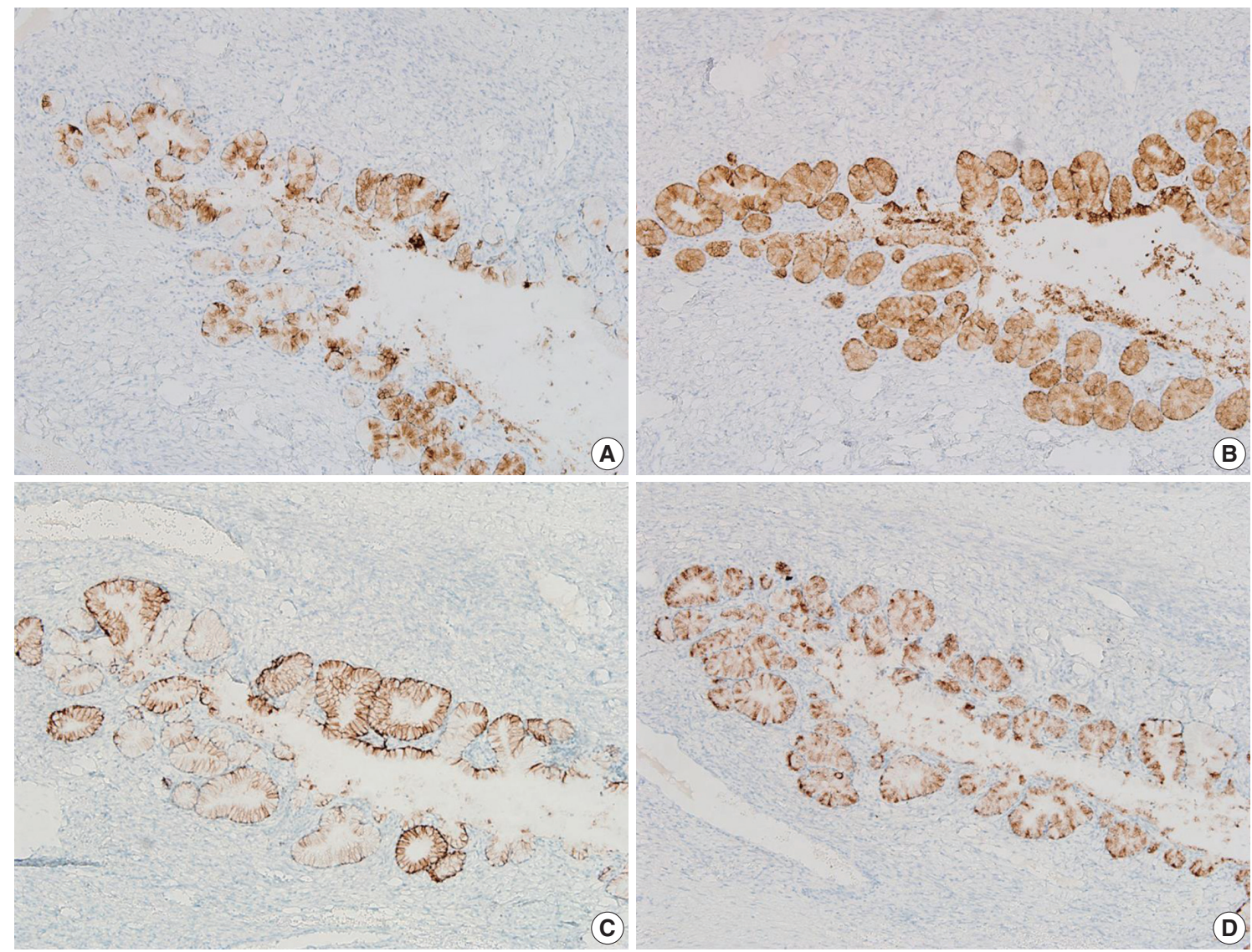

Fig. 2. The mucinous epithelium of the ovary shows immunoreactivity for various gastric markers, including MUC5AC (A), MUC6 (B), carbonic anhydrase-IX (C), and HIK1083 (D), indicating the gastric phenotype of the mucinous epithelium.

surgery due to intussusception of the small bowel of unknown cause. During the preoperative evaluation, colonoscopy identified eight hamartomatous polyps, nine hyperplastic polyps, and two tubulovillous adenomas in her large intestine. She had abundant mucocutaneous melanin pigmentation around the lips. Based on her clinical features and the histopathological diagnosis of hamartomatous polyps and mucocutaneous pigmentation, she was clinically diagnosed with PJS. No other family members had clinical signs of PJS. On pelvic and abdominal ultrasonography and computed tomography, she was found to have huge bilateral ovarian cystic masses with ascites in the pelvic cavity. On exploratory laparotomy, the left ovarian mass was ruptured, and approximately $1,500 \mathrm{~mL}$ of ascitic fluid was identified. The patient underwent hysterectomy, bilateral salpingo-oophorectomy, and small bowel polypectomy via small bowel enterostomy.

Grossly, the left ovarian tumor was a multilocular cyst measuring $22 \times 20 \times 6 \mathrm{~cm}$ (Fig. 1A), whereas the right ovarian tumor was an oligolocular cyst measuring $8 \times 7 \times 5 \mathrm{~cm}$ (Fig. 1B). The septa of the cysts were thin and the internal surface was smooth. Both cystic tumors contained mucinous fluid without any solid
Table 1. PCR primers for the STK11 gene

\begin{tabular}{lll}
\hline STK11 exon & \multicolumn{1}{c}{ Sense $\left(5^{\prime}\right.$ to $\left.3^{\prime}\right)$} & \multicolumn{1}{c}{ Antisense $\left(5^{\prime}\right.$ to $\left.3^{\prime}\right)$} \\
\hline Exon 1a & tggagaagggaagtcggaa & tgaggatcttgacggccc \\
Exon 1b & tcggcaagtacctgatggg & gaaagtccgtaacgcaggc \\
Exon 2 & gtacgccacttccacaggg & ggaaccagggaaggccac \\
Exon 3 & cctccagagccccttttct & gcttgcagtgagccgagat \\
Exon 4 & ctgtggtgtttgggaggct & agaaggtgtccaggccgtt \\
Exon 5 & ggggaacctgctgctcac & tgtggccagagagggtctg \\
Exon 6 & cctctggtccagcagcca & tcggcctctccactcagtc \\
Exon 7 & ccagggectgacaacagag & actgcccagagaccccact \\
Exon 8 & ctcctgagtgtgtggcaggt & caccagagggcagaagctg \\
Exon 9 & cagctgtaagtgcgtcccc & ccatgactgactagcgcgg \\
\hline
\end{tabular}

$\mathrm{PCR}$, polymerase chain reaction.

area. Microscopically, both ovarian tumors showed cysts of varying sizes lined by a single layer of columnar epithelium, and the architecture of the glandular arrangement was characterized by clusters of small glands budding from surrounding a centrally located large duct-like structures, forming a lobular arrangement at lower magnification (Fig. 1C). The mucinous epithelium had pale eosinophilic cytoplasm with basally located nuclei (Fig. 1D). Neither epithelial stratification nor nuclear atypia 
was identified. There were no foci of sex cord tumor with annular tubules in both ovaries. The uterine cervix, endometrium, and fallopian tubes were meticulously examined, but no histologic evidence of mucinous epithelial lesions or metaplasia was identified.

Polyps of the small intestine showed a branching polypoid

A

90100110120130140150160170180190200210220230240250260270280290300310320330340350360370380390
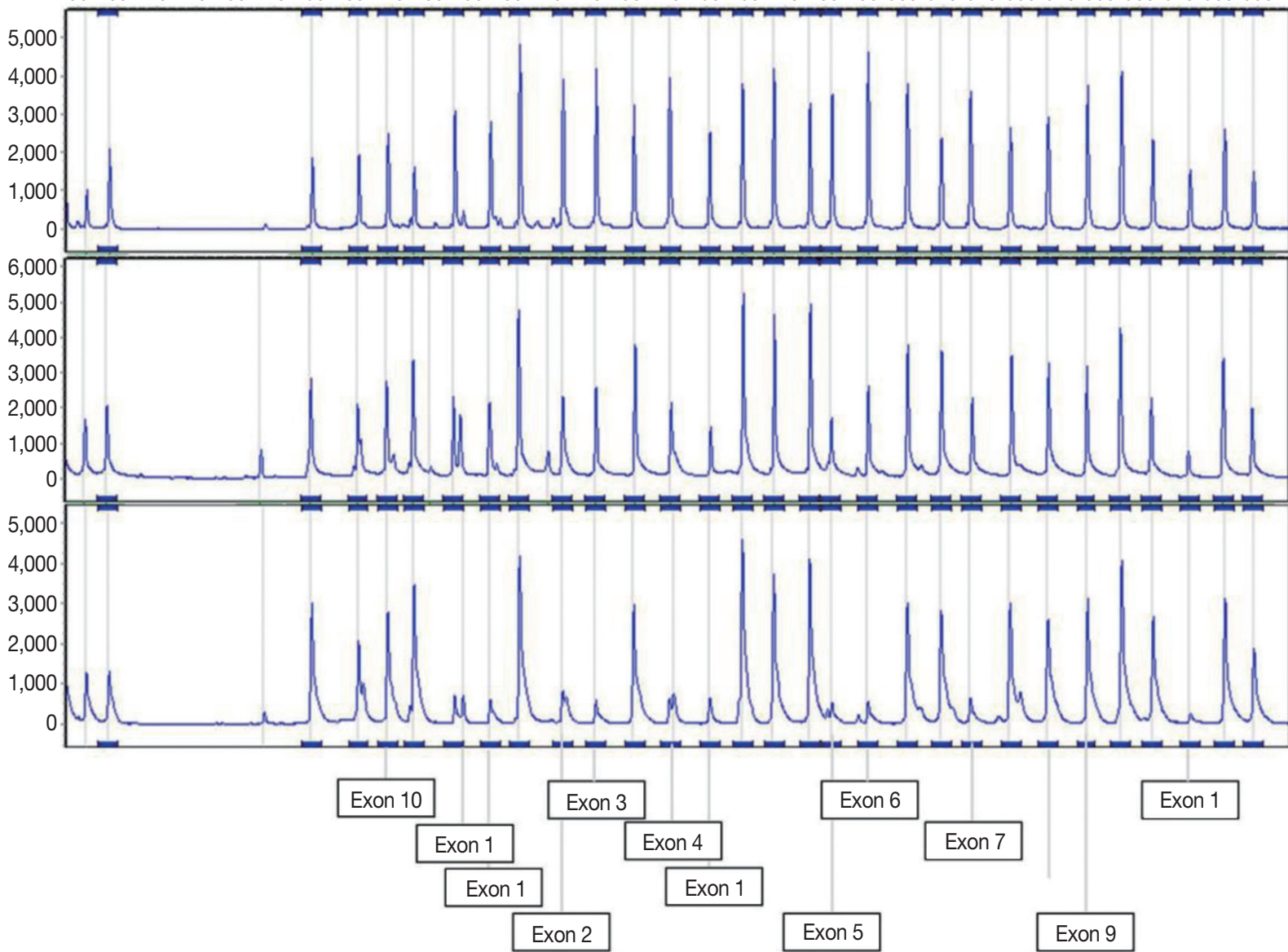

B

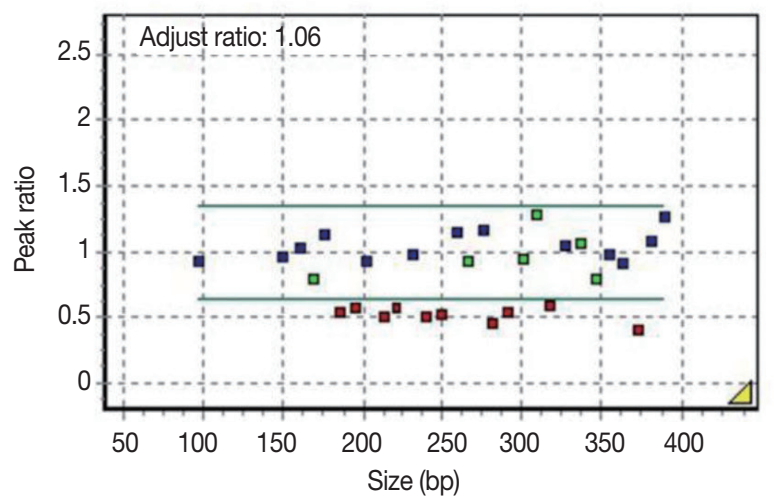

C

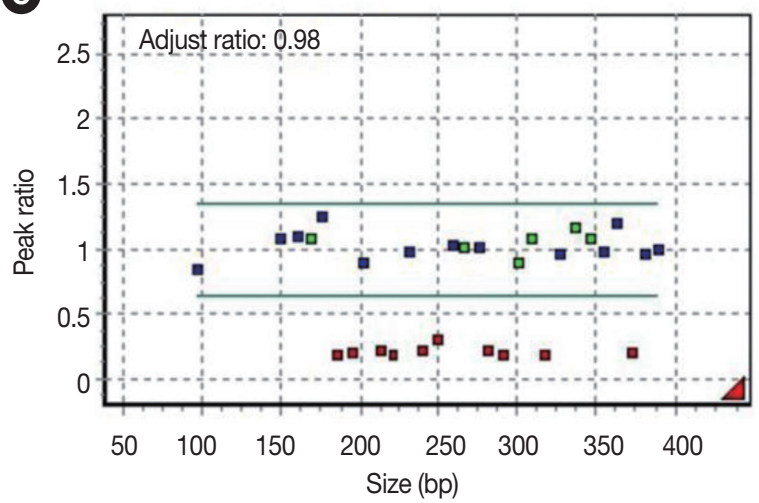

Fig. 3. (A) Electrogram showing multiple ligation-dependent probe amplification analysis of the STK11 gene. The X-axis indicates product size (bp) and the Y-axis indicates the fluorescence intensities as the dosage of the products. The exon numbers of STK11 are marked below the electrogram. The peaks are those of a normal control (A, upper), peripheral blood leukocyte (A, middle), and left ovarian mucinous cystadenoma (A, lower). (B) In the peripheral blood leukocytes, a peak ratio less than 0.65 represented the deletion mutation in the STK11 gene. (C) Ovarian mucinous tumor with lobular endocervical glandular hyperplasia-like features showing mosaic loss of heterozygosity on mutation analysis (heteroplasmy) through exon 1 to 7 with a peak ratio of about 0.25 . The $X$-axis indicates product size (bp) and the $Y$-axis represents the adjusted peak ratios with normal controls. The adjusted ratio was standardized to the control sample, and the median point was considered to be 1.0 . 
structure with crypts and villi of variable lengths and cystically dilated glands, which were divided by muscularis mucosa branching in various directions (Fig. 1E), as highlighted by desmin immunostaining (Fig. 1F). On combined Alcian blue ( $\mathrm{pH}$ 2.5) and periodic acid-Schiff after diastase (DPAS) staining, the intracytoplasmic mucin in the epithelium of the ovarian tumor was negative for Alcian blue $\mathrm{pH} 2.5$ and positive for DPAS. The cytoplasm stained bright pink for combined Alcian blue $\mathrm{pH}$ 2.5/DPAS, implying that the mucinous contents of the ovarian tumor were neutral (Fig. 1G), in contrast to mucin in the normal endocervical mucosa, which was positive for both Alcian blue $\mathrm{pH} 2.5$ and DPAS and showed a purple-violet color for combined Alcian blue pH2.5/DPAS (Fig. 1G, inlet).

\section{Immunohistochemistry}

We performed immunohistochemical staining using formalin-fixed, paraffin-embedded, 4 - $\mu$ m-thick tissue sections with an OptiView DAB immunohistochemical detection kit (Roche Diagnostics, Mannheim, Germany) on a Benchmark XT autoimmunostainer (Ventana Medical System, Tucson, AZ, USA). The mucinous epithelium of the ovary showed diffuse immunoreactivity for MUC6 (Novo, Newcastle upon Tyne, UK), moderately intense immunoreactivity for carbonic anhydrase-IX (Novus Biologicals, Littleton, CO, USA), and focal immunoreactivity for MUC5AC (marker of foveolar-type mucin; Novo) and HIK1083 (TOYO 2CHOME, Tokyo, Japan) (Fig. 2A-D). The epithelium did not show immunoreactivity for p16 (Santa Cruz Biotechnology, Santa Cruz, CA, USA), estrogen receptor (Novo), progesterone receptor (Novo), or p53 (Oncogene, Uniondale, NY, USA). The Ki-67 labelling index was less than $5 \%$.

\section{Mutation analysis}

Genomic DNA was extracted from peripheral blood samples by a QIAamp DNA blood kit (Qiagen GmbH, Hilden, Germany) and from fresh frozen tissue of the left ovarian tumor by a QuickGene DNA tissue kit (Fujifilm Life Science, Tokyo, Japan). The STK11 gene was amplified via polymerase chain reaction by using 10 sets of primers in intronic flanking regions containing all exons (Table 1). Sequencing analysis was performed by a cycle sequencing kit (Thermo Fisher Scientific, Waltham, MA, USA) following the manufacturer's instructions.

Multiple ligation-dependent probe amplification (MLPA) was performed to detect deletions and duplications in the STK11 gene by STK11 MLPA kit P101 (MRC-Holland, Amsterdam, The Netherlands). The peak height of the probes was analyzed using GeneMarker software v.1.7 (SoftGenetics LLC, State Col- lege, PA, USA). A peak ratio less than 0.65 was interpreted as a 'deletion,' whereas a peak ratio greater than 1.35 was interpreted as a 'duplication.'

Sequencing analysis did not detect any mutation in the exons and exon-intron boundaries of the STK11 gene in the blood and ovarian tumor samples. Using MLPA analyses, however, peripheral blood leukocytes showed a germline heterozygous deletion mutation at exons 1-7 of the STK11 gene (c.1-?_920+?del) and the tumor tissue showed mosaic loss of heterozygosity due to the mutation (heteroplasmy) (Fig. 3A-C).

\section{DISCUSSION}

Minimal deviation adenocarcinoma (an extremely well differentiated form of gastric type adenocarcinoma) of the cervix and ovarian sex cord tumor with annular tubules are the most wellknown tumors of the female genital organs associated with PJS, but association with ovarian mucinous tumors has rarely been described. ${ }^{4}$ Moreover, gastric phenotype of the mucinous epithelium in patients with PJS has not been clearly determined, and there is only one case briefly mentioning ovarian mucinous tumor with pyloric/gastric differentiation. ${ }^{4}$ In that case report, the pyloric gland differentiation involved the lesions of multiple organs, including the jejunum, urinary bladder, uterine cervix, fallopian tube, and ovary. In the extragenital organs, Peutz-Jeghers polyps in duodenum and jejunum have small areas of normal superficial gastric-type epithelium, suggesting that the proliferations of gastric-type mucinous epithelium are common features in any organs of the patients with PJS. Although the gastric subtype is very rare among mucinous tumors, it must be a distinct subtype of the ovarian mucinous tumors, which should be differentiated from the usual intestinal subtype.

LEGH in the uterine cervix is thought to be a precursor lesion of gastric-type cervical adenocarcinoma, ${ }^{5}$ which is often identified in patients with PJS. Therefore, the premalignant potential of an ovarian mucinous tumor resembling LEGH should be closely monitored.

In our case, a germline heterozygous deletion mutation in STK11 (c.1-? 920+?del) (exon1-7del) and mosaic loss of heterozygosity were detected in the left ovarian mucinous cystadenoma by MLPA assay, but direct sequencing did not detect the deletion of the large exon.

In conclusion, our case combined with previously described cases suggests that STK11 gene mutations play an important role in the proliferation of pyloric gland-phenotype mucinous epithelium in various anatomical locations. 


\section{Conflicts of Interest}

No potential conflict of interest relevant to this article was reported.

\section{Acknowledgments}

The tissue used in this study was provided by the Asan BioResource Center, Korea-Biobank Network (number 2015$03[93])$.

\section{REFERENCES}

1. Ferry JA, Young RH, Engel G, Scully RE. Oxyphilic Sertoli cell tumor of the ovary: a report of three cases, two in patients with the Peutz-Jeghers syndrome. Int J Gynecol Pathol 1994; 13: 259-66.
2. Jenne DE, Reimann H, Nezu J, et al. Peutz-Jeghers syndrome is caused by mutations in a novel serine threonine kinase. Nat Genet 1998; 18: 38-43.

3. Kuragaki C, Enomoto T, Ueno Y, et al. Mutations in the STK11 gene characterize minimal deviation adenocarcinoma of the uterine cervix. Lab Invest 2003; 83: 35-45.

4. Kato N, Sugawara M, Maeda K, Hosoya N, Motoyama T. Pyloric gland metaplasia/differentiation in multiple organ systems in a patient with Peutz-Jegher's syndrome. Pathol Int 2011; 61: 369-72.

5. Kondo T, Hashi A, Murata S, et al. Endocervical adenocarcinomas associated with lobular endocervical glandular hyperplasia: a report of four cases with histochemical and immunohistochemical analyses. Mod Pathol 2005; 18: 1199-210. 